\title{
The Need for a Neutral Speaking Period in Psychosocial Stress Testing
}

\author{
Sarah J. Grimley, ${ }^{1,2}$ Celine M. Ko, ${ }^{2}$ Holly E. R. Morrell, ${ }^{3}$ Fran Grace, ${ }^{4}$ Maria S. Bañuelos, ${ }^{1}$ \\ Brandon R. Bautista, ${ }^{1}$ Gilbert N. Chavez, ${ }^{1}$ Emily R. Dalrymple, ${ }^{1}$ Matthew Green, ${ }^{1,2}$ \\ Jessica Gurning, ${ }^{1}$ Anne C. Heuerman, ${ }^{1,4}$ Misael Huerta, ${ }^{1}$ Megan Marks, ${ }^{1}$ Jenny Ov, ${ }^{1}$ \\ Petter Overton-Harris, ${ }^{1}$ and Lisa E. Olson ${ }^{1}$
}

${ }^{1}$ Department of Biology, University of Redlands, CA, USA

${ }^{2}$ Department of Psychology, University of Redlands, CA, USA

${ }^{3}$ Department of Psychology, Loma Linda University, Loma Linda, CA, USA

${ }^{4}$ Department of Religious Studies, University of Redlands, CA, USA

\begin{abstract}
Tasks such as the Trier Social Stress Test, narrative recall, and some cognitive challenges require participants to speak in order to measure acute physiological responses to induced stress. Typically, the physiological measures during the stressed state are compared to a silent baseline period. This does not differentiate between stress that is induced by emotion and stress due to the physical act of vocalization. We modified a psychosocial stress task for 41 participants to add a period of neutral speaking. We hypothesized that there would be significant differences in physiological measures between the silent baseline and neutral speaking periods, and that these differences would explain a substantial proportion of the stress response traditionally attributed to emotion. Blood pressure, skin conductance level, respiration rate, salivary alpha-amylase, and high frequency heart rate variability showed significant changes during the neutral speaking period compared to a silent baseline, demonstrating the need for this control. Of the magnitude of physiological response which would have typically been attributed to emotion, 36-77\% was due to vocalization alone. In stress-inducing tasks that require speaking, care should be taken in study design to account for the physiological impact of speech.
\end{abstract}

Keywords: stress, speech, blood pressure, skin conductance, heart rate variability

Various standardized protocols have been used to induce emotional and physiological stress in the laboratory. The cold pressor test, presentation of video clips, cognitive challenges like the Stroop task, and the Trier Social Stress Test (TSST; Bali \& Jaggi, 2015) are routinely employed to capture the participants' physiological response to a stressful stimulus. Typically, the physiological measures during the stressed state are compared to a silent, resting baseline period. The magnitude of the difference between these periods is considered the stress reactivity.

Many laboratory stressor tasks require the participant to speak, particularly those that rely on a social component to enhance stress induction. For example, personal narrative, such as describing an angering event from the past, is performed aloud to an interviewer (Kop et al., 2011; Lawler-Row, Karremans, Scott, Edlis-Matityahou, \& Edwards, 2008; Maisto, Ewart, Connors, Funderburk, \& Krenek, 2009). Mental arithmetic is also spoken to allow the researcher to assess whether answers are correct (McFadden, Healy, Hoversten, Ito, \& Hernandez, 2012;
Noto, Sato, Kudo, Kurata, \& Hirota, 2005). One portion of the TSST, a "gold-standard" social-evaluative threat task originally developed to evaluate the hypothalamus-pituitary-adrenal axis but now used to assess wide-ranging physiological responses, requires participants to make a speech on why they should be hired for a mock employment position (Kirschbaum, Pirke, \& Hellhammer, 1993). This speech has been modified to suit the needs of various studies, such as describing views of interpersonal violence and related Spanish legislation (Romero-Martinez, Nunes-Costa, Lila, Gonzalez-Bono, \& Moya-Albiol, 2014), defending oneself from an accusation of shoplifting (Buchanan, Bagley, Stansfield, \& Preston, 2012), or role play regarding an auto accident (Smith \& Jordan, 2015), but all require verbalization.

However, when such stressor tasks are compared to a silent baseline period, it is impossible to differentiate between stress due to emotion/anxiety and stress due to the physical act of vocalization. Speaking itself does alter physiological measures, including heart rate (Seraganian, Szabo, \& Brown, 1997), heart rate variability 
(Bernardi et al., 2000; Sloan, Korten, \& Myers, 1991), blood pressure (Linden, 1991), and skin conductance (Crane, Dieker, \& Brown, 1970; Tomaka, Blascovich, \& Swart, 1994). Although a few attempts have been made to create a control task for the TSST (Het, Rohleder, Schoofs, Kirschbaum, \& Wolf, 2009; Wiemers, Schoofs, \& Wolf, 2013), these protocols are time- and staff-intensive, and these studies evaluated limited autonomic markers. The majority of the literature on the stress response still does not account for the effect of simply speaking aloud on physiological arousal.

Here, we propose a novel modification to a psychosocial stress protocol which can be implemented with little additional time or resources. By adding a period of neutral speech, we were able to quantify the effect of pure vocalization on a variety of autonomic stress responses and compare it to emotion-based responses. We hypothesized that there would be significant differences in physiological measures between the silent baseline and neutral speaking periods, and that these differences would explain some or all of the stress response traditionally attributed to emotion.

\section{Materials and Methods}

\section{Participants}

This study was approved by the University of Redlands Institutional Review Board (IRB Approval \#2014-26R3REDLANDS), and participants gave written informed consent. Participants were undergraduate students recruited for a larger study (Ko et al., in press) assessing the impact of a meditation-based seminar on compassion by means of university-wide advertising via flyer. Students who were interested in participating in the seminar were randomized to either the semester-long course $(n=21)$ or a wait-list control group $(n=20)$. For the purposes of this report, the control and intervention groups were analyzed together (see Statistical Analysis; $n=41$ total; 14 males and 27 females). There was not a difference in distribution of genders between participants randomized to the course versus the control group. Participants were between the ages of 18 and 24 ( $M=19.8 \pm 1.4$ years), proficient in English, did not have severe mental health issues such as active psychosis, did not have substantial previous meditation experience, were not pregnant, and were not using anxiolytic medications or tobacco. Participants who completed an assessment both prior to and following the seminar were compensated $\$ 50$; those who completed only the first assessment were compensated $\$ 20$. Thirty-four participants returned for the second assessment. There was no association between gender and whether or not participants returned for the second assessment. For most analyses, the first and second assessments were combined for this report (see Statistical Analysis).

After giving informed consent, participants completed psychological measures including the State-Trait Anxiety Inventory (STAI; Spielberger \& Gorsuch, 1983), the Center for Epidemiologic Studies Depression Scale (CES-D), and the ten-question Perceived Stress Scale (PSS-10). The STAI is an indicator of the severity of both state and trait anxiety measured by two subscales (STAI-S \& STAI-T). Scores range from 20 to 80 , with higher scores indicating greater anxiety. The CES-D has been used in both clinical and nonclinical populations to screen for clinical levels of depression. Scores range from 0 to 60 , with higher scores indicating more severe symptoms of depression (Radloff, 1977; Shean \& Baldwin, 2008). The PSS-10 measures the degree to which individuals appraise situations in their lives as stressful. Scores range from 0 to 40 , with higher scores indicating more perceived stress (Cohen, Kamarck, \& Mermelstein, 1983; Cohen \& Williamson, 1988).

\section{Stress Induction}

Participants were tested between 14:00 and 17:00, having been awake for a minimum of $6 \mathrm{hr}$ prior to their appointment. Participants were asked not to drink any alcohol in the $24 \mathrm{hr}$ prior to the experiment, and not to eat, drink, exercise, or brush their teeth in the hour prior to testing. Participants were also instructed to drink caffeine according to their normal pattern of caffeine intake. Participants were sitting throughout the test, and two researchers were present during data collection.

The entire stress induction took less than $30 \mathrm{~min}$ (Figure 1). The data collection protocol began with a baseline period of participants sitting quietly for 5 min while listening to calming sounds (Calmsound, Seaside Ocean Sounds from a Golden Sand Beach.Mp3, Seaside 4). A 5-minute neutral questioning period followed the baseline period and consisted of asking participants questions that were not intended to be stressful. This portion of the test served as a comparative baseline measurement to account for the effect of vocalization on the stress response. Some questions were recommended as neutral control questions by the manufacturer of our skin conductance amplifier (iWorx, Inc., Dover, NH); other similar questions were added to extend the neutral questioning period to $5 \mathrm{~min}$. The neutral questions utilized in this study were asked in the same order for each participant:

(1) Do you live in a dormitory?

(2) How many pets do you have?

(3) How many siblings do you have?

(4) Were you born in the United States?

(5) What year are you? (e.g., freshman) 
Continuous monitoring: Skin conductance level, heart rate variability, respiration rate

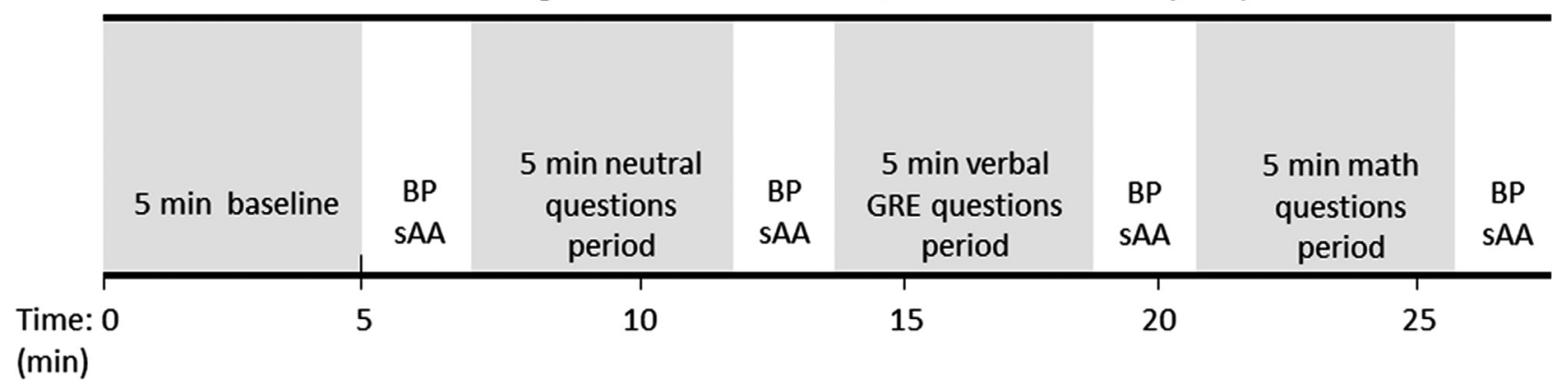

Figure 1. Timeline of stress induction. Approximately 2 min elapsed between periods which were excluded from analysis of skin conductance level, heart rate variability, and respiration rate. $\mathrm{BP}=$ blood pressure measurement was taken; $\mathrm{SAA}$ = saliva sample was obtained for later analysis of salivary alpha-amylase; GRE = Graduate Record Examination.

(6) What is your major?

(7) Do you like chocolate?

(8) Do you like broccoli?

(9) Do you have a car?

(10) Do you like the beach?

(11) Where are you from?

(12) What is your favorite food?

(13) What is your favorite animal?

(14) How old are you?

(15) Do you play a sport?

(16) Do you play an instrument?

(17) Do you have a roommate?

(18) Have you traveled outside of the country?

(19) What is your favorite color?

(20) Do you have a bicycle on campus?

(21) Do you prefer more dressy or more casual clothes?

(22) Have you ever been to Texas?

(23) What day of the week is today?

(24) Have you gone to the movies in the past month?

(25) Are you right or left handed?

Questions were asked at a slow pace and not all participants finished all 25 questions. The average number answered within 5 min was $17 \pm 2.8$ questions.

The stressor periods consisted of verbal and math questioning. During the verbal questioning period, participants were asked a series of analogy questions taken from a previous Graduate Record Examination (GRE) exam for 5 min. This portion was included for a larger study in order to simulate an academic stressor. Participants were told after each question whether their answer was correct or incorrect. Questions were never repeated for the same participant. A math questioning period followed in which participants were asked to perform a serial subtraction task for $5 \mathrm{~min}$. Participants serially subtracted 13 starting from the number 1,022 (or 1,033 on the second assessment) as quickly and accurately as possible. After every mistake, participants were asked to stop and start over from 1,022.

\section{Physiological Measures}

\section{Blood Pressure}

An Omron BP7911T monitor with Com Fit Cuff (Omron Healthcare, Inc., Lake Forest, IL) was placed on the upper left arm for measurement of systolic and diastolic blood pressure. One measurement was taken at the end of each 5-minute period.

\section{Skin Conductance Level}

Two circular $\mathrm{Ag} / \mathrm{AgCl}$ electrodes with $8 \mathrm{~mm}$ diameter contact area were secured with Velcro straps on the volar surface of the distal second (index) and fourth (ring) fingers of the left hand for measurement of the tonic electrodermal activity, recorded as skin conductance level. The GSR200 amplifier (iWorx Systems, Inc., Dover, NH) applied constant $200 \mathrm{mV}$ for exosomatic recording of current. Data were captured using the 12-bit resolution IX-214 Four Channel Recorder with a sampling rate of 100 samples/s using the program Labscribe 2 (iWorx Systems, Inc.). Vivosense software (Vivosense 2.6, Vivonetics, San Diego, CA) was used to apply a low-pass filter $(0.0555 \mathrm{~Hz})$ to remove phasic skin conductance responses and calculate the average of skin conductance level for each period. Each of the four time periods (baseline, neutral questioning, verbal questioning, and math questioning) were defined on the continuous recording in Vivosense using the annotation tool. The first minute of the baseline period was excluded from analysis to allow the participant to acclimate to the equipment and laboratory, and thus the baseline average was calculated on a 4-minute recording. For the latter three periods, the average was based on a 5-minute recording. 


\section{Heart Rate Variability}

A WristOx ${ }_{2}{ }^{\text {TM }}$ Model 3150 wrist-worn pulse oximeter (Nonin Medical Inc., Plymouth, MN) was placed on the wrist and right index finger to capture a continuous pulse plethysmograph. The pulse oximeter transmitted via Bluetooth to the Nonin software. These data were imported into Vivosense software (Vivosense 2.6, Vivonetics, San Diego, $\mathrm{CA})$. Baseline, neutral questioning, verbal questioning, and math questioning time periods were annotated as described for skin conductance. The Artifact Management feature in Vivosense as well as manual editing were used to identify and exclude abnormalities in pulse plethysmograph data. Vivosense software applied a power spectral transformation on the tachogram to calculate the power of each frequency component. The high frequency range was defined as $0.15 \mathrm{~Hz}-0.4 \mathrm{~Hz}$. Normalized high frequency power (high frequency power/total power) was calculated to estimate parasympathetic activity.

\section{Respiration Rate}

A respiration monitor strap was placed around the chest to detect motion (iWorx Systems, Inc., Dover, NH). Data were captured using the IX-214 Four Channel Recorder with a sampling rate of 100 samples/s using the program Labscribe 2 (iWorx Systems, Inc.). These data were imported into Vivosense software (Vivosense 2.6, Vivonetics, San Diego, CA). Baseline, neutral questioning, verbal questioning, and math questioning time periods were annotated as described for skin conductance. Average respiration rate for each period was calculated using the breath detection algorithm in Vivosense which uses minimum tidal volume of $48 \mathrm{ml}$.

\section{Salivary Alpha-Amylase}

A passive saliva sample was taken via an oral swab under the tongue (Salimetrics, State College, PA) immediately following each of the four time periods. Saliva samples were stored at $-20{ }^{\circ} \mathrm{C}$ and later analyzed using the salivary alpha-amylase kinetic enzyme assay kit (Salimetrics, State College, $\mathrm{PA})$. In this assay, salivary alpha-amylase catalyzes the conversion of a chromogenic substrate (2-chloro-p-nitrophenol linked with maltotriose) to a yellow-colored product (2chloro-p-nitrophenol) which absorbs $405 \mathrm{~nm}$ light. Salivary alpha-amylase enzyme activity is proportional to the increase in absorbance over time. The AgileReader ${ }^{\mathrm{TM}}$ ELISA Plate Reader (ACTgene, Inc., Piscataway, NJ) was used for the kinetic measurements. The average coefficient of variation on inter-assay replicates was $9.6 \%$.

\section{Statistical Analysis}

All statistical analyses were performed using IBM SPSS statistical software (International Business Machines Corporation, Armonk, NY). Psychological measure scores were compared to the normative means by running one-sample $t$-tests. Correlations between respiration rate and other physiological measures were tested using Pearson's product-moment correlation.

For physiological dependent variables, we initially conducted 3-way analysis of variances (ANOVAs) with $\alpha$ levels of .05. These $2 \times 2 \times 4$ ANOVAs tested the effect of randomization (between subjects: compassion seminar vs. wait-list control), assessment time (within subjects: preseminar vs. post-seminar), and period (within subjects: baseline, neutral questioning, verbal questioning, math questioning). Data were examined for violations of normality and assumptions of ANOVA prior to analysis. When necessary, data were transformed by taking $\log _{10}$; in these cases, the conclusions were the same for ANOVAs performed on transformed and non-transformed data, and therefore results are presented for the non-transformed data set. For violations of sphericity, the GreenhouseGeisser or Huynh-Feldt corrections were used appropriately based on epsilon values. For respiration, systolic blood pressure, diastolic blood pressure, skin conductance level, and alpha-amylase, there were no main effects of randomization or assessment time, and no interaction effects which would have indicated an effect of the seminar (all $p s>.05)$. Therefore data were collapsed across randomization and assessment time for analysis of the main effect of period. For normalized high frequency heart rate variability power, other main and interaction effects were present therefore relevant data are presented here from the first assessment only. Where statistically significant differences were found for the main effect of period, post hoc pairwise comparisons were conducted. A Bonferroni correction yielded an $\alpha$ of .008 due to multiple comparisons. Caffeine intake was initially used as a covariate but did not change any conclusions, and therefore data are presented here without the use of the covariate.

The percent of the peak stress response that can be attributed to vocalization alone was calculated utilizing the formula: |(value at neutral questioning period - value at baseline)/(value at peak stressor period - value at baseline) $\mid \times 100$. The peak stressor period was determined to be either the verbal questioning period or the math questioning period by evaluation of which had the greater difference from baseline.

The sample size of 41 participants was reduced in different ways depending on the analysis (see each figure legend for relevant $n$ ). Seven participants did not return for the second assessment; two participants' blood pressure readings and one participant's skin conductance level were excluded for being outliers; three participants' heart rate variability could not be analyzed because of excessive motion artifacts; and four participants' respiration data were not recorded due to an equipment failure on one day of testing. 
Table 1. Psychological measure scores $(M \pm S D)$ of the sample compared to normative values from (Herman et al., 2011; Roberti et al., 2006; Spielberger \& Gorsuch, 1983)

\begin{tabular}{lcccc}
\hline & & \multicolumn{2}{c}{ Normative values } \\
\cline { 3 - 5 } & Men $(n=14)$ & Women $(n=27)$ & Men & Women \\
\hline STAI-S & $34.9 \pm 10.3$ & $38.8 \pm 9.6$ & $36.5 \pm 10.0$ & $38.8 \pm 12.0$ \\
STAI-T & $38.6 \pm 13.0$ & $42.6 \pm 14.3$ & $38.3 \pm 9.2$ & $40.4 \pm 10.2$ \\
CES-D $^{a}$ & $15.5 \pm 9.9$ & $16.3 \pm 6.8$ & $12.9 \pm 8.5$ & $15.4 \pm 9.7$ \\
PSS-10 & $14.6 \pm 5.8$ & $17.7 \pm 7.6$ & $17.4 \pm 6.1$ & $18.4 \pm 6.5$ \\
\hline
\end{tabular}

Notes. STAI-S = State-Trait Anxiety Inventory, State subscale; STAI-T = State-Trait Anxiety Inventory, Trait subscale; CES-D = Center for Epidemiologic Studies Depression Scale; PSS-10 = Perceived Stress Scale. aMeans reported with one outlier removed (see text).

The collection of salivary samples after each period was a late addition to the protocol and data at all four time points is only available for 12 participants; this data should be interpreted cautiously due to the limited sample size.

\section{Results}

Our sample of university students had scores similar to normative values (Herman et al., 2011; Roberti, Harrington, \& Storch, 2006; Spielberger \& Gorsuch, 1983) for state and trait anxiety (STAI-S and STAI-T), depressive symptoms (CES-D), and perceived stress (PSS-10) ( $p$ s > .05; Table 1). The CES-D means exclude one outlier scoring 52 out of 60 (severe depressive symptoms). The exclusion of this outlier did not change the conclusion that CES-D scores were not statistically different than the normative value.

To determine whether the physiological indicators of the "emotional stress response" can actually be explained by participants simply speaking aloud, we determined whether there were statistically significant differences observed between the baseline and neutral questioning time periods, and whether the magnitude of these differences fully or partially accounted for the magnitude of the differences between baseline and the stressor periods. A main effect of period was found for systolic blood pressure, $\left.F(3,90)=1,377.6, p<.001, \eta_{\mathrm{p}}{ }^{2}=0.521\right)$ as well as diastolic blood pressure, $F(3,90)=29.177, p<.001, \eta_{\mathrm{p}}{ }^{2}=0.493$ (Figures $2 \mathrm{~A}$ and $2 \mathrm{~B})$. The act of speaking neutrally contributed $42.4 \%$ of the magnitude of the peak stress response in systolic blood pressure, and $40.3 \%$ of the diastolic response. Similarly, a main effect of period was found for skin conductance level, $F(1.657,51.360)=48.922, p<.001$, $\eta_{\mathrm{p}}{ }^{2}=0.612$ (Figure 2C). For this physiological measure, $62.9 \%$ of the peak stress response was accounted for by neutral speech. The analysis of salivary alpha-amylase as a marker of sympathetic nervous system activity was a late addition to our methodology, so the sample size is limited $(n=12)$ and the data should be interpreted cautiously. However, even within this subset, a main effect of period was evident, $F(3,30)=4.714, p<.01, \eta_{\mathrm{p}}{ }^{2}=0.320$ (Figure 2D). The act of speaking neutrally contributed $35.8 \%$ of the magnitude of the peak alpha-amylase stress response. Normalized high frequency power of heart rate variability, as a measure of parasympathetic tone, also varied by period, $F\left(1.9995,70.385=6.904, p<.01, \eta_{\mathrm{p}}{ }^{2}=0.161\right.$ (Figure 3). Neutral speech alone accounted for $77.6 \%$ of the maximum drop in parasympathetic tone.

In order to see if these findings could be explained by changes in respiration rate between periods, we conducted a similar analysis of breaths per minute. Although a main effect of period was evident for respiration rate, $F(2.002$, $54.048)=28.240, p<.001, \eta_{\mathrm{p}}{ }^{2}=0.511$ ), with neutral speaking accounting for $76.8 \%$ of the peak response, the pattern of post hoc testing was not the same as the other physiological measures (Figure 4). Unlike other physiological measures, the neutral questions period is statistically different from the verbal questions period, but neither is statistically different from the math questions period. Additionally, the respiration rate did not correlate to any other physiological measure ( $r$ values between -0.22 and $0.04 ; p s>.05)$.

\section{Discussion}

The vast majority of the literature utilizing psychosocial stress testing does not account for the effect of vocalization in experimental procedures. Consistent with our hypothesis, we have shown that physiological measures such as blood pressure, skin conductance level, salivary alphaamylase, and heart rate variability are significantly different between a neutral speaking period and a silent baseline. The common reporting of the change from a silent baseline to a stressor period as being the "stress response" is likely to be inaccurate when measuring the autonomic nervous system, as we have demonstrated that $36-78 \%$ of such a response can be attributed to vocalization alone. Although some attention has been given to measure the effect of speech on cortisol responses in the classic TSST protocol, this is less true for autonomic responses; in other commonly used psychosocial stressors (narrative recall, cognitive tasks like the Stroop test), investigators routinely do not account for vocalization at all.

The TSST was originally developed to induce a cortisol response (Kirschbaum et al., 1993), and it has been validated that cortisol does not rise due to vocalization using controls similar to our neutral speaking period. In the "friendly" TSST (Wiemers et al., 2013), participants are asked to speak about their life and career aspirations and then to read 30 words aloud to a panel of encouraging 
(A)

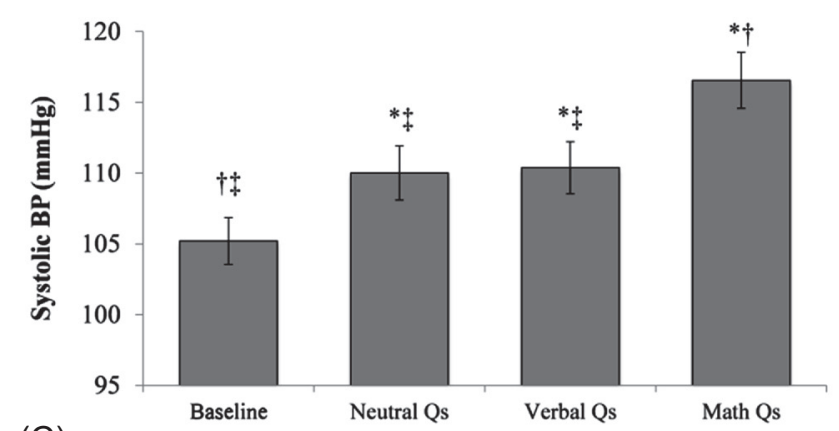

(C)

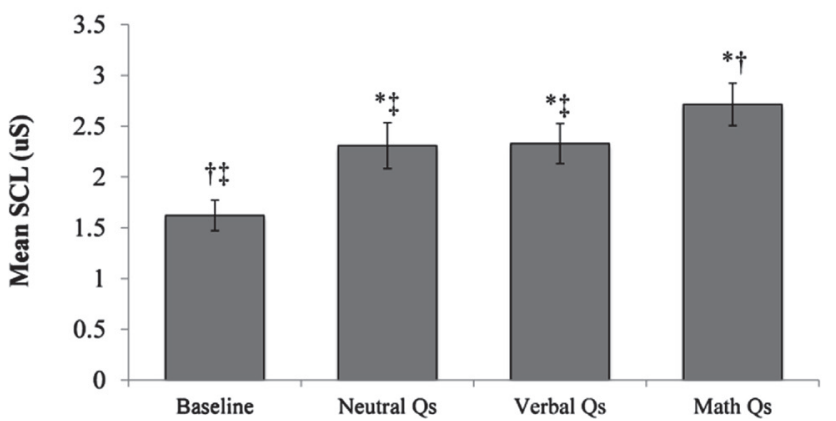

(B)

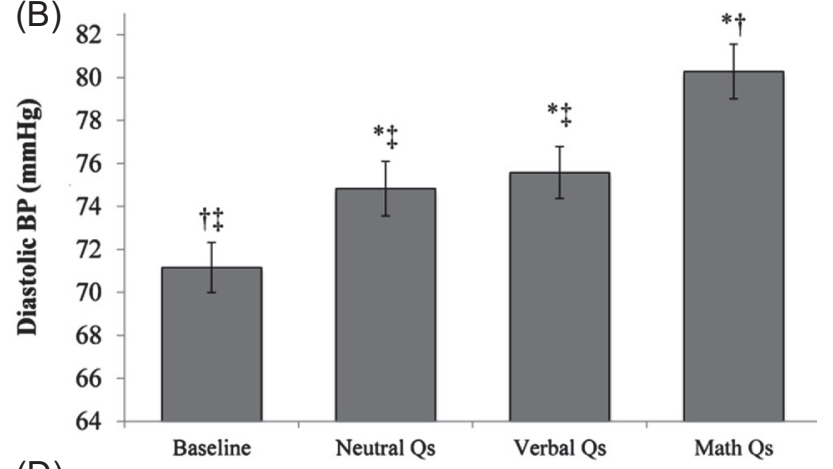

(D)

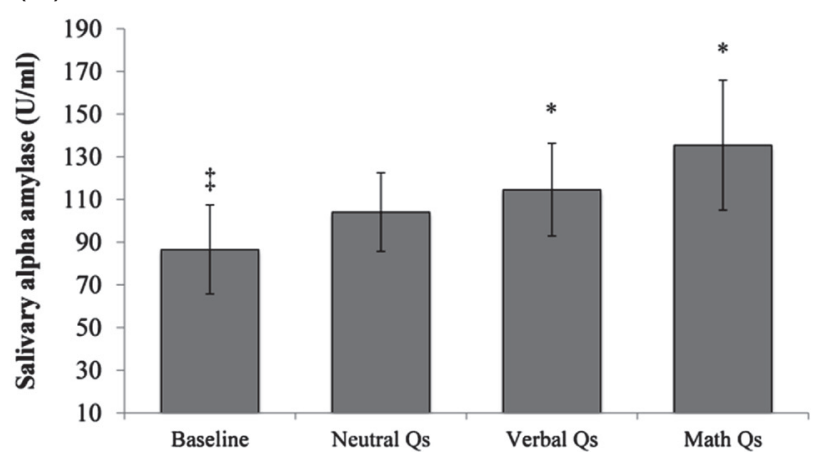

Figure 2. Measures of the sympathetic nervous system in each period of a psychosocial stress test. (A) Systolic blood pressure (BP), $n=32$; (B) Diastolic blood pressure (BP), $n=32$; (C), Skin conductance level (SCL), $n=33$; and (D) Salivary alpha-amylase, $n=12$, were measured for baseline, neutral questions, verbal questions, and math questions (Qs), with the latter two periods intended as stressors. *Different from baseline $(p<.005)$; †different from neutral questioning period $(p<.005)$; different from math questioning period $(p<.005)$. Error bars represent standard error.

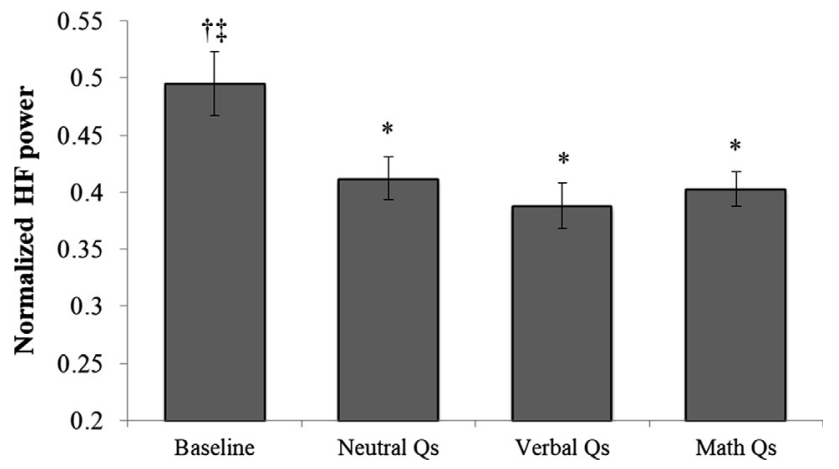

Figure 3. Normalized high frequency (HF) power of heart rate variability in each period of a psychosocial stress test. High frequency power is indicative of parasympathetic activity, thus a drop is expected due to stress. *Different from baseline $(p<.005) ; \dagger$ different from neutral questioning period $(p<.005)$; $\$$ different from math questioning period $(p<.005)$. Error bars represent standard error; $n=38$. Qs = questions.

listeners. In the "placebo" TSST (Het et al., 2009), participants are asked to discuss a movie, book, or recent trip and perform simple addition in an empty room. A combination of reading aloud and then counting or simple arithmetic has also been used (Almela et al., 2011; von Dawans, Kirschbaum, \& Heinrichs, 2011), or simply reading aloud

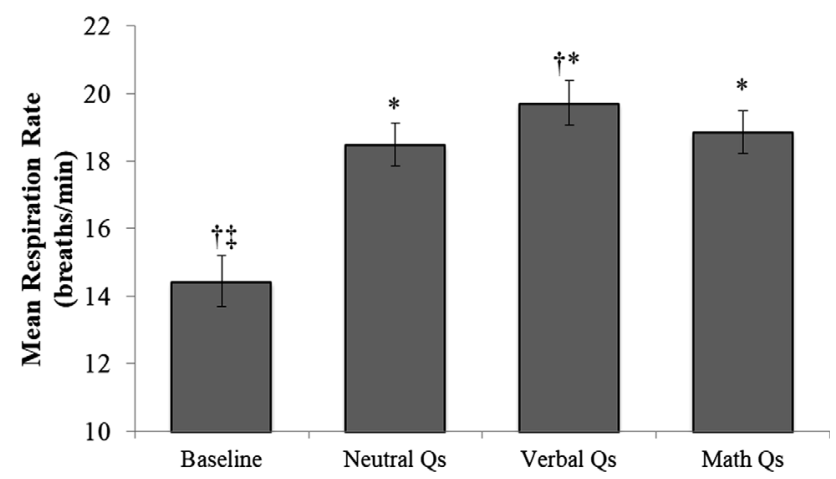

Figure 4. Mean respiration rate of four periods of a psychosocial stress test. *Different from baseline $(p<.005)$; †different from neutral questioning period $(p<.005)$; $\$$ different from math questioning period $(p<.005)$. Unlike other physiological measures, the neutral questions period is statistically different from the verbal questions period, but neither is statistically different from the math questions period. Error bars represent standard error; $n=30$. Qs = questions.

(Rohleder, Wolf, Maldonado, \& Kirschbaum, 2006). This work has established that the cortisol response in the TSST is emotion-induced, as it is robustly distinguishable from cortisol levels due to the simple act of speech. These protocols are valuable but time and resource intensive, as they have been implemented with a complete second session 
with the mock stressor, or a separate control group. This effectively doubles either the time required to conduct the experiment or the sample size required. We propose that the inclusion of one additional 5-minute period into an existing protocol is a more cost-effective and simple method to control for the effects of vocalization on measures of the stress response. Ideally, in future studies, such a period could be counterbalanced with the stressor period for even more robust measurements.

In contrast to cortisol measurements, the vocalization effect in the TSST on the autonomic nervous system has not been broadly assessed. This is regrettable because the original and modified versions of the TSST have often been used to induce sympathetic responses in addition to hypothalamus-pituitary-adrenal markers (Allen, Kennedy, Cryan, Dinan, \& Clarke, 2014). The findings on the effect of neutral speech on the sympathetic nervous system in the TSST are mixed, and mostly limited to salivary alpha-amylase and heart rate. The alpha-amylase response to the placebo TSST (in one of two experiments) or friendly version of the TSST was indistinguishable from the traditional TSST (Het et al., 2009; Wiemers et al., 2013). Reading aloud with counting generated approximately $1 / 3-1 / 2$ of the peak stress TSST stress response (Almela et al., 2011), and reading alone did not elevate salivary alpha-amylase at all (Rohleder et al., 2006). Heart rate rose approximately $1 / 3-1 / 2$ of the peak stress response due to reading and counting (Almela et al., 2011; von Dawans et al., 2011), and reading alone caused an equivalent heart rate and heart rate variability response to the TSST (Rohleder et al., 2006). Previous authors (Het et al., 2009) have suggested that future studies explore expanded indicators of autonomic activity, which we have done here by assessing blood pressure, skin conductance level, and heart rate variability in addition to alpha-amylase. Combined with previous work, our data point to an autonomic nervous system response to stressors that is largely due to the act of speech rather than emotion.

An assertion could be made that the response we observed during neutral speech was not due to vocalization, but due to the novelty of the laboratory environment or anticipation of the stressors to come. However, the silent baseline period captures exactly these factors. Our baseline occurred in the same room and in the presence of the same researchers as during the stress period, and participants were aware of the protocol since the baseline occurred after informed consent. Thus, the inclusion of both the silent baseline and the neutral speaking periods allows a differentiation of anticipatory/novelty stress versus vocalization.

Our protocol is not intended to simply be an addition the TSST (in fact, we were not modeling our experiment on the classic TSST protocol with a committee), but is relevant to any psychosocial stressor that involves speech such as verbal arithmetic, narrative recall, or cognitive tasks. We propose that care should be taken to develop an emotionally neutral speaking control that matches the stressor task as much as possible. In reviewing previous work (Almela et al., 2011; von Dawans et al., 2011; Rohleder et al., 2006) we felt it was not ideal to introduce a new variable into a control task such as reading. Reading requires different cognitive resources than other tasks (Cabeza \& Nyberg, 2000). It has been shown that reading aloud and spontaneous speech can have distinct timing characteristics (Bóna, 2014; Wang, Green, Nip, Kent, \& Kent, 2010) and fundamental frequency (Hudson \& Holbrook, 1982) that could influence physiological parameters. We propose that no control task will be perfect, but attention should be given to match cognitive modality with neutral emotional content as much as possible.

This leads to the difficult question of what is truly neutral content. We considered that some of our questions might provoke an emotional response from participants (e.g., asking if a participant has a pet could be upsetting if that person's pet just died), but we anticipated that such an event would not affect the majority of participants. An additional concern was that a study examining the influence of specific types of words on the physiological stress response found that personal words, such as a participant's major in college, produced greater skin conductance than either pleasant words, such as love, or aversive words, such as cancer (Crane et al., 1970). Since skin conductance level was captured continuously and we marked when each question was asked on the data file, we were able to analyze the mean conductance level following each individual question. No questions elicited higher conductance than others across the sample (data not shown), increasing our confidence that we chose questions that were usually neutral.

The changes we observed due to speech in blood pressure, skin conductance level, alpha-amylase, and heart rate variability were not correlated to respiration rate, so the effects are not due to simply a difference in breathing during speaking versus silence. Other factors, such as muscle tension or vibration of vocal cords (Hansen \& Patil, 2007), could be explored in the future to understand this effect more fully. Elucidating factors such as these may help explain why subjective emotional stress often does not correlate to physiological responses in laboratory testing (Campbell \& Ehlert, 2012).

It would be useful for a future study to explore if these results are similar in different populations. Our participants were limited to young adult college students. It is possible that older participants may feel more socially comfortable answering neutral questions in a laboratory environment, and thus the effect of neutral speech would be smaller. Alternatively, students may be accustomed to being asked a variety of questions by an authority figure due to regular experience in the classroom. Non-students may have a different response. It is also possible that the gender of 
the researcher could affect the perceived stressfulness of these tasks. Gender of the participant was not a statistically significant factor in our study except for systolic blood pressure, where men overall had higher values than women (data not shown); in all physiological measures, both genders showed the same pattern of change due to period. However, evaluating the interaction effect of gender of the participant and gender of the researcher would be a valuable inquiry. Since our experimental design included two researchers in the room with the participant, our sample size is not large enough to analyze all possible gender combinations with sufficient statistical power.

We believe these results have valuable implications for the field of physiological measurement of induced stress. It is not surprising that speaking induces sympathetic activation, and yet many experimental designs only use a silent baseline control. Multiple studies have used psychosocial stress testing to examine whether interventions, such as stress management, self-compassion training, meditation, or acupressure, were successful in reducing participants' emotional stress response from pre- to post-intervention (Arch et al., 2014; Kemeny et al., 2012; McFadden et al., 2012; Rose et al., 2013; Vocks, Ockenfels, Jürgensen, Mussgay, \& Rüddel, 2004). Without accounting for the effect of speech, it is not possible to determine whether an emotional stress response was induced in their participants in the first place, and how much that emotional response was mitigated versus the simple stress of vocalization.

\section{Ethics and Disclosure Statements}

All participants of the study provided written informed consent and the study was approved by the Institutional Review Board.

All authors disclose no actual or potential conflicts of interest including any financial, personal, or other relationships with other people or organizations that could inappropriately influence (bias) their work.

\section{Acknowledgment}

This work was supported by the Trust for the Meditation Process and the University of Redlands.

\section{References}

Allen, A. P., Kennedy, P. J., Cryan, J. F., Dinan, T. G., \& Clarke, G. (2014). Biological and psychological markers of stress in humans: Focus on the Trier Social Stress Test. Neuroscience and Biobehavioral Reviews, 38, 94-124. https://doi.org/ 10.1016/j.neubiorev.2013.11.005

Almela, M., Hidalgo, V., Villada, C., van der Meij, L., Espin, L., Gomez-Amor, J., \& Salvador, A. (2011). Salivary alpha-amylase response to acute psychosocial stress: The impact of age. Biological Psychology, 87, 421-429. https://doi.org/10.1016/j. biopsycho.2011.05.008
Arch, J. J., Brown, K. W., Dean, D. J., Landy, L. N., Brown, K. D., \& Laudenslager, M. L. (2014). Self-compassion training modulates alpha-amylase, heart rate variability, and subjective responses to social evaluative threat in women. Psychoneuroendocrinology, 42, 49-58. https://doi.org/10.1016/j.psyneuen.2013.12.018

Bali, A., \& Jaggi, A. S. (2015). Clinical experimental stress studies: Methods and assessment. Reviews in the Neurosciences, 26, 555-579. https://doi.org/10.1515/revneuro-2015-0004

Bernardi, L., Wdowczyk-Szulc, J., Valenti, C., Castoldi, S., Passino, C., Spadacini, G., \& Sleight, P. (2000). Effects of controlled breathing, mental activity and mental stress with or without verbalization on heart rate variability. Journal of the American College of Cardiology, 35, 1462-1469. https://doi.org/10.1016/ S07351097(00)00595-7

Bóna, J. (2014). Temporal characteristics of speech: The effect of age and speech style. Journal of the Acoustical Society of America, 136, EL116-EL121. https://doi.org/10.1121/1.4885482

Buchanan, T. W., Bagley, S. L., Stansfield, R. B., \& Preston, S. D. (2012). The empathic, physiological resonance of stress. Social Neuroscience, 7, 191-201. https://doi.org/10.1080/ 17470919.2011 .588723

Cabeza, R., \& Nyberg, L. (2000). Imaging cognition II: An empirical review of 275 PET and fMRI studies. Journal of Cognitive Neuroscience, 12, 1-47. https://doi.org/10.1162/089892900511 37585

Campbell, J., \& Ehlert, U. (2012). Acute psychosocial stress: Does the emotional stress response correspond with physiological responses? Psychoneuroendocrinology, 37, 1111-1134. https://doi.org/10.1016/j.psyneuen.2011.12.010

Cohen, S., Kamarck, T., \& Mermelstein, R. (1983). A global measure of perceived stress. Journal of Health and Social Behavior, 24, 385-396. https://doi.org/10.2307/2136404

Cohen, S., \& Williamson, G. (1988). Perceived stress in a probability sample of the United States. In S. Spacapan \& S. Oskamp (Eds.), The Social Psychology of Health: The Claremont Symposium on Applied Social Psychology (pp. 31-67). Thousand Oaks, CA: Sage.

Crane, L. D., Dieker, R. J., \& Brown, C. T. (1970). The physiological response to the communication modes: Reading, listening, writing, speaking, and evaluating. Journal of Communication, 20, 231-240. https://doi.org/10.1111/j.1460-2466.1970.tb00882.x

Hansen, J. H., \& Patil, S. (2007). Speech under stress: Analysis, modeling and recognition. In C. Müller (Ed.), Speaker classification I: Fundamentals, features and methods (pp. 108-137). Berlin, Germany: Springer.

Herman, S., Archambeau, O. G., Deliramich, A. N., Kim, B. S., Chiu, P. H., \& Frueh, B. C. (2011). Depressive symptoms and mental health treatment in an ethnoracially diverse college student sample. Journal of American College Health, 59, 715-720. https://doi.org/10.1080/07448481.2010.529625

Het, S., Rohleder, N., Schoofs, D., Kirschbaum, C., \& Wolf, O. T. (2009). Neuroendocrine and psychometric evaluation of a placebo version of the "Trier Social Stress Test". Psychoneuroendocrinology, 34, 1075-1086. https://doi.org/10.1016/j. psyneuen.2009.02.008

Hudson, A. I., \& Holbrook, A. (1982). Fundamental frequency characteristics of young black adults: Spontaneous speaking and oral reading. Journal of Speech, Language, and Hearing Research, 25, 25-28. https://doi.org/10.1044/jshr.2501.25

Kemeny, M. E., Foltz, C., Cavanagh, J. F., Cullen, M., Giese-Davis, J., Jennings, P., ... Ekman, P. (2012). Contemplative/emotion training reduces negative emotional behavior and promotes prosocial responses. Emotion, 12, 338-350. https://doi.org/ 10.1037/a0026118

Kirschbaum, C., Pirke, K.-M., \& Hellhammer, D. H. (1993). The "Trier Social Stress Test" - A tool for investigating psychobiological 
stress responses in a laboratory setting. Neuropsychobiology, 28, 76-81. https://doi.org/10.1159/000119004

Ko, C. M., Grace, F., Chavez, G. N., Grimley, S. J., Dalrymple, E. R., \& Olson, L. E. (in press). Effect of seminar on compassion on student self-compassion, mindfulness and well-being: A randomized controlled trial. Journal of American College Health. https://doi.org/10.1080/07448481.2018.1431913

Kop, W. J., Synowski, S. J., Newell, M. E., Schmidt, L. A., Waldstein, S. R., \& Fox, N. A. (2011). Autonomic nervous system reactivity to positive and negative mood induction: The role of acute psychological responses and frontal electrocortical activity. Biological Psychology, 86, 230-238. https://doi.org/ 10.1016/j.biopsycho.2010.12.003

Lawler-Row, K. A., Karremans, J. C., Scott, C., Edlis-Matityahou, M., \& Edwards, L. (2008). Forgiveness, physiological reactivity and health: The role of anger. International Journal of Psychophysiology, 68, 51-58. https://doi.org/10.1016/j.ijpsycho. 2008.01.001

Linden, W. (1991). What do arithmetic stress tests measure? Protocol variations and cardiovascular responses. Psychophysiology, 28, 91-102. https://doi.org/10.1111/j.1469-8986.1991. tb03393.x

Maisto, S. A., Ewart, C. K., Connors, G. J., Funderburk, J. S., \& Krenek, M. (2009). Use of the social competence interview and the anger transcendence challenge in individuals with alcohol use disorder. Journal of Behavioral Medicine, 32, 285-293. https://doi.org/10.1007/s10865-009-9201-z

McFadden, K. L., Healy, K. M., Hoversten, K. P., Ito, T. A., \& Hernandez, T. D. (2012). Efficacy of acupressure for nonpharmacological stress reduction in college students. Complementary Therapies in Medicine, 20, 175-182. https://doi.org/ 10.1016/j.ctim.2011.12.003

Noto, Y., Sato, T., Kudo, M., Kurata, K., \& Hirota, K. (2005). The relationship between salivary biomarkers and state-trait anxiety inventory score under mental arithmetic stress: A pilot study. Anesthesia and Analgesia, 101, 1873-1876. https://doi. org/10.1213/01.ANE.0000184196.60838.8D

Radloff, L. S. (1977). The CES-D scale. Applied Psychological Measurement, 1, 385-401. https://doi.org/10.1177/ 014662167700100306

Roberti, J. W., Harrington, L. N., \& Storch, E. A. (2006). Further psychometric support for the 10 item version of the perceived stress scale. Journal of College Counseling, 9, 135-147. https://doi.org/10.1002/j.2161-1882.2006.tb00100.x

Rohleder, N., Wolf, J. M., Maldonado, E. F., \& Kirschbaum, C. (2006). The psychosocial stress-induced increase in salivary alpha-amylase is independent of saliva flow rate. Psychophysiology, 43, 645-652. https://doi.org/10.1111/j.1469-8986.2006. 00457.x

Romero-Martinez, A., Nunes-Costa, R., Lila, M., Gonzalez-Bono, E., \& Moya-Albiol, L. (2014). Cardiovascular reactivity to a marital conflict version of the Trier Social Stress Test in intimate partner violence perpetrators. Stress, 17, 321-327. https://doi.org/10.3109/10253890.2014.919448

Rose, R. D., Buckey, J. C. Jr., Zbozinek, T. D., Motivala, S. J., Glenn, D. E., Cartreine, J. A., \& Craske, M. G. (2013). A randomized controlled trial of a self-guided, multimedia, stress management and resilience training program. Behaviour
Research and Therapy, 51, 106-112. https://doi.org/10.1016/j. brat.2012.11.003

Seraganian, P., Szabo, A., \& Brown, T. G. (1997). The effect of vocalization on the heart rate response to mental arithmetic. Physiology \& Behavior, 62, 221-224. https://doi.org/10.1016/ S0031-9384(97)00102-9

Shean, G., \& Baldwin, G. (2008). Sensitivity and specificity of depression questionnaires in a college-age sample. The Journal of Genetic Psychology, 169, 281-292. https://doi.org/10.3200/ GNTP.169.3.281-292

Sloan, R., Korten, J., \& Myers, M. M. (1991). Components of heart rate reactivity during mental arithmetic with and without speaking. Physiology \& Behavior, 50, 1039-1045. https://doi. org/10.1016/0031-9384(91)90434-P

Smith, T. W., \& Jordan, K. D. (2015). Interpersonal motives and social-evaluative threat: Effects of acceptance and status stressors on cardiovascular reactivity and salivary cortisol response. Psychophysiology, 52, 269-276. https://doi.org/ 10.1111/psyp.12318

Spielberger, C., \& Gorsuch, R. (1983). State-trait anxiety inventory for adults: Manual and sample: Manual, instrument and scoring guide. Palo Alto, CA: Consulting Psychologists Press.

Tomaka, J., Blascovich, J., \& Swart, L. (1994). Effects of vocalization on cardiovascular and electrodermal responses during mental arithmetic. International Journal of Psychophysiology, 18, 23-33. https://doi.org/10.1016/0167-8760(84)90012-6

Vocks, S., Ockenfels, M., Jürgensen, R., Mussgay, L., \& Rüddel, H. (2004). Blood pressure reactivity can be reduced by a cognitive behavioral stress management program. International Journal of Behavioral Medicine, 11, 63-70. https://doi.org/10.1207/ s15327558ijbm1102_1

von Dawans, B., Kirschbaum, C., \& Heinrichs, M. (2011). The Trier Social Stress Test for Groups (TSST-G): A new research tool for controlled simultaneous social stress exposure in a group format. Psychoneuroendocrinology, 36, 514-522. https://doi. org/10.1016/j.psyneuen.2010.08.004

Wang, Y. T., Green, J. R., Nip, I. S., Kent, R. D., \& Kent, J. F. (2010). Breath group analysis for reading and spontaneous speech in healthy adults. Folia Phoniatrica et Logopedica, 62, 297-302. https://doi.org/10.1159/000316976

Wiemers, U. S., Schoofs, D., \& Wolf, O. T. (2013). A friendly version of the Trier social stress test does not activate the HPA axis in healthy men and women. Stress, 16, 254-260. https://doi.org/ $10.3109 / 10253890.2012 .714427$

Received March 29, 2017

Revision received January 8, 2018

Accepted April 23, 2018

Published online July 18, 2018

\section{Lisa E. Olson}

1200 E. Colton Ave.

Redlands

CA 92373

USA

lisa_olson@redlands.edu 